\title{
Cholinergic Interneurons Control the Excitatory Input to the Striatum
}

\author{
Pavel Pakhotin and Enrico Bracci \\ Faculty of Life Sciences, University of Manchester, Manchester M60 1QD, United Kingdom
}

\begin{abstract}
How the extent and time course of presynaptic inhibition depend on the action potentials of the neuron controlling the terminals is unknown. We investigated this issue in the striatum using paired recordings from cholinergic interneurons and projection neurons. Glutamatergic EPSCs were evoked in projection neurons and cholinergic interneurons by stimulation of afferent fibers in the cortex and the striatum, respectively. A single spike in a cholinergic interneuron caused significant depression of the evoked glutamatergic EPSC in $34 \%$ of projection neurons located within $100 \mu \mathrm{m}$ and $41 \%$ of cholinergic interneurons located within $200 \mu \mathrm{m}$. The time course of these effects was similar in the two cases, with EPSC inhibition peaking 20-30 ms after the spike and disappearing after 40-80 ms. Maximal depression of EPSC amplitude was up to $27 \%$ in projection neurons and to $19 \%$ in cholinergic interneurons. These effects were reversibly blocked by muscarinic receptor antagonists (atropine or methoctramine), which also significantly increased baseline EPSC (evoked without a preceding spike in the cholinergic interneuron), suggesting that some tonic cholinergic presynaptic inhibition was present. This was confirmed by the fact that lowering extracellular potassium, which silenced spontaneously active cholinergic interneurons, also increased baseline EPSC amplitude, and these effects were occluded by previous application of muscarinic receptor antagonists. Collectively, these results show that a single spike in a cholinergic interneuron exerts a fast and powerful inhibitory control over the glutamatergic input to striatal neurons.
\end{abstract}

Key words: acetylcholine; ACh; basal ganglia; glutamate; interneurons; presynaptic mechanisms; striatum

\section{Introduction}

Presynaptic inhibition is a major form of communication in the CNS (Nicoll et al., 1990; Wu and Saggau, 1997; Turecek and Trussell, 2001). Crucial insight into this phenomenon has come from single intracellular recordings and application of exogenous agonists. These experiments, however, do not allow one to assess the effects of endogenously released transmitters and specifically how the extent and time course of presynaptic inhibition depend on the firing activity of the neurons that release the neurotransmitter responsible for these effects. Paired intracellular recordings have been widely used to study how direct synaptic transmission between two cells depends on individual action potentials in the presynaptic neurons (Miles and Poncer, 1996). Here we show that paired recordings also provide an experimental tool to study presynaptic inhibition. This is achieved by electrically activating a certain set of fibers impinging on the recorded neurons and investigating how action potentials in one of the neurons affect the synaptic responses of the other cell. We used this approach to investigate how cholinergic interneurons in the striatum affect the glutamatergic input to projection neurons and to other cholinergic interneurons.

The striatum is the main input nucleus of the basal ganglia and

Received Aug. 25, 2006; revised Dec. 4, 2006; accepted Dec. 4, 2006 This work was funded by Wellcome Trust Project Grant 071943.

Correspondence should be addressed to Dr. Enrico Bracci, Faculty of Life Sciences, University of Manchester, Manchester M60 1 QD, UK. E-mail: e.bracci@manchester.ac.uk.

D01:10.1523/JNEUROSCI.3709-06.2007

Copyright $\odot 2007$ Society for Neuroscience $\quad$ 0270-6474/07/270391-10\$15.00/0 is extremely rich in acetylcholine and cholinergic receptors (Aubert et al., 1996; Contant et al., 1996; Aznavour et al., 2002). Striatal acetylcholine, and its interplay with dopamine, have long been recognized to play a crucial role in voluntary movement (Duvoi$\sin , 1967)$. In the striatum, acetylcholine is mainly released by a population of large, aspiny interneurons (LAIs) (Phelps et al., 1985; Zhou et al., 2002; Tepper and Bolam, 2004). These interneurons correspond to the tonically active neurons recorded in the striatum in vivo that respond to rewarding and salient stimuli (Cragg, 2006).

A widespread excitatory input from the cerebral cortex impinge on striatal medium spiny projection neurons (MSNs), which in turn send GABAergic projections to other basal ganglia nuclei. Understanding the dynamics of corticostriatal information processing is one of the main challenges in the basal ganglia field. LAIs receive their main fast excitatory synaptic input from the thalamus, although a smaller cortical projection is also present (Lapper and Bolam, 1992; Reynolds and Wickens, 2004). They are therefore in the position to mediate complex feedforward and feedback actions in the basal ganglia. Consistent with the high density of acetylcholine markers, a number of presynaptic and postsynaptic neuronal mechanisms are controlled by nicotinic and muscarinic acetylcholine receptors in the striatum (Calabresi et al., 2000; Zhou et al., 2003). At presynaptic level, it has been shown that the glutamatergic input to MSNs is inhibited by activation of $\mathrm{M}_{2}$ and $\mathrm{M}_{3}$ muscarinic receptors (Malenka and Kocsis, 1988; Sugita et al., 1991; Calabresi et al., 1998b; Hernandez-Echeagaray et al., 1998). Based on this evidence, we 
investigated how individual action potentials generated by LAIs affect the glutamatergic synaptic input to MSNs and other LAIs.

\section{Materials and Methods}

Slice preparation. Wistar rats (14-24 d postnatal, both sexes) were killed by cervical dislocation in accordance with the United Kingdom Animals Act of 1986; oblique brain slices (300-400 $\mu \mathrm{m}$ thick) were quickly dissected using a vibroslicer (Camden Instruments, Loughborough, UK) and maintained at $25^{\circ} \mathrm{C}$ in oxygenated artificial CSF (ACSF) (in mM: 126 $\mathrm{NaCl}, 2.5 \mathrm{KCl}, 1.3 \mathrm{MgCl}_{2}, 1.2 \mathrm{NaH}_{2} \mathrm{PO}_{4}, 2.4 \mathrm{CaCl}_{2}, 10$ glucose, and 18 $\mathrm{NaHCO}_{3}$ ). Low potassium solution was obtained by lowering the ACSF $\mathrm{KCl}$ concentration from 2.5 to $0.5 \mathrm{~mm}$, without additional compensation.

Oblique slices were cut in a plane rotated by $45^{\circ}$ with respect to the horizontal one to preserve part of the corticostriatal connections intact, as described by Kawaguchi et al. (1989). For recordings, slices were submerged, superfused $(2-3 \mathrm{ml} / \mathrm{min})$ at $25^{\circ} \mathrm{C}$, and visualized with a $40 \times$ water-immersion objective (Olympus Optical, Tokyo, Japan) using standard infrared and differential interference contrast microscopy.

Whole-cell recordings. Paired, whole-cell recordings were performed with $1.5 \mathrm{~mm}$ external diameter borosilicate pipettes, filled with intracellular solution (in mM: $125 \mathrm{~K}$-gluconate, $15 \mathrm{KCl}, 0.04$ EGTA, 12 HEPES, 2 $\mathrm{MgCl}_{2}, 4 \mathrm{Na}_{2} \mathrm{ATP}$, and $0.4 \mathrm{Na}_{2} \mathrm{GTP}$, adjusted to $\mathrm{pH} 7.3$ with $\mathrm{KOH}$ ). With this solution, pipette resistance was $3-5 \mathrm{M} \Omega$. Recordings in fast currentclamp or voltage-clamp mode were performed with an EPC10 double amplifier and Patchmaster acquisition software (HEKA Elektronik, Lambrecht/Pfalz, Germany). Data were acquired at $10-15 \mathrm{kHz}$. Fast and slow capacitance compensations were used. For voltage-clamp recordings, the uncompensated series resistance was $<20 \mathrm{M} \Omega$ during all of the experiments that were accepted for analysis. Drugs were bath applied through the superfusion system. Methoctramine tetrahydrochloride hemihydrate, atropine, muscarine chloride, and neostigmine bromide were obtained from Sigma (Poole, UK). Recorded neurons were usually located in the dorsorostral portion of the striatum because, in this area, the synaptic responses evoked by stimulation of the cortex (see below) tended to be larger. The distance between two neurons was measured as the minimal distance between the somata of the cells as visible with the optics used. This is therefore less than the distance between the centers of the cells, which we did not attempt to measure, because it was not unequivocally defined under the present conditions.

Evoked synaptic responses. Synaptic responses in the MSNs were elicited with a bipolar electrode placed in the medial agranular cortex, close to the white matter between the striatum and the cortex (see Fig. $1 A$ ). Stimulation amplitude was 2-50 V, and its duration was $0.1-0.5 \mathrm{~ms}$. These MSN responses, recorded under either current-clamp (as EPSPs) or voltage-clamp (as EPSCs) conditions, were completely blocked by coapplication of the ionotropic glutamate receptor blockers 2,3dihydroxy-6-nitro-7-sulfonyl-benzo[f] quinoxaline (NBQX) $\left(\begin{array}{lll}10 & \mu \mathrm{M}\end{array}\right)$ and AP-5 $(10 \mu \mathrm{M})(n=12)$, confirming that they resulted solely from activation of glutamatergic afferents. To elicit robust glutamatergic responses in LAIs, the stimulating electrode had usually to be placed within the striatum (see Fig. $1 B$ ). In fact, stimulation of the cortex or white matter was often ineffective in eliciting detectable responses in LAIs (see example in Fig. 6A). This is consistent with the fact that LAIs receive glutamatergic inputs mainly from the thalamus (Lapper and Bolam, 1992). To avoid stimulation of local striatal neurons and fibers, the stimulating electrode was placed at distances $>600 \mu \mathrm{m}$ from the recorded neurons. These responses, recorded under either current- or voltageclamp conditions were completely abolished by coapplication of NBQX and AP-5 $(n=11)$, showing that they were entirely mediated by ionotropic glutamate receptors. In most cases, the evoked synaptic responses were considerably larger (in amplitude and duration) in one of the two recorded LAIs. The neuron with larger synaptic responses was then voltage clamped at $-60 \mathrm{mV}$, while spikes were evoked in the other cell.

In both cases, a single electrical stimulus was applied every $15 \mathrm{~s}$, and the first five responses were not used for analysis. This frequency was chosen because, after the fifth stimulus, the EPSC amplitude did not display a statistically significant trend of variation as a function of time (linear least-squares fittings had always a slope $<0.005 \mathrm{mV} / \mathrm{s}$, never significantly different from zero; $p>0.4$, bootstrap test), even when the stimulation was applied for $>10 \mathrm{~min}(n=9)$. This kind of analysis was described previously (Bracci and Panzeri, 2006).

Spike-EPSC intervals. We evoked synaptic responses at different intervals after a spike was elicited in the LAI. Such spikes were triggered under current-clamp conditions by short (10 ms) but relatively large (150-350 $\mathrm{pA}$ ) current injections. If spontaneously active, LAIs were slightly hyperpolarized (at approximately $-60 \mathrm{mV}$ ) throughout the experiment to prevent spontaneous spikes and to allow us to precisely time spikes with current injections. The intervals were measured from the peak of the spike to the peak of the synaptic response (EPSC or EPSP), because this temporal distance was well defined in all cases and was significant from a biological point of view. The values tested (which were defined as positive if the spike preceded the EPSC) were $-10,0,10,20,30,40,60,80$, and $100 \mathrm{~ms}$. In each pair, the protocol was repeated at least 10 times for each interval tested (and for the case with no LAI spike). A certain scattering of the experimentally observed intervals around the desired value was inevitable. However, it was always possible to obtain intervals that were within $2.5 \mathrm{~ms}$ from the desired one. Therefore, for the analysis, the intervals were grouped into $5 \mathrm{~ms}$ bins centered on the intervals mentioned before.

Statistical analysis. Values are expressed as mean $\pm \mathrm{SD}$, and statistical comparisons between EPSC or EPSP amplitudes measured in the absence or presence of a spike in an LAI were made using Student's $t$ test for unpaired data. To compare the effects of atropine and methoctramine, the EPSC amplitudes with or without spike in the LAI were normalized to their baseline value (i.e., in control solution with no spike in the LAI) and the Kolmogorov-Smirnov (K-S) test for two data sample was used. The $\mathrm{K}-\mathrm{S}$ test was also used when comparing ratios of two EPSC amplitudes (i.e., baseline EPSC vs EPSC in the presence of a spike in an LAI), because the assumption of normal distribution was not tenable in this case. Two populations of data points were considered to be significantly different if $p<0.05$.

\section{Results}

Current- and voltage-clamp whole-cell recordings were obtained from MSNs and LAIs. LAIs were identified thanks to their large somata (Bolam et al., 1984; Kawaguchi, 1993) and their distinctive electrophysiological properties, which included (1) a depolarizing sag that developed during a hyperpolarizing step of current, (2) a rebound depolarization observed after the end of a hyperpolarizing step, and (3) marked, long-lasting afterpolarizations after bursts of spikes (Kawaguchi, 1993; Blomeley and Bracci, 2005; Wilson, 2005). These features are illustrated in the example of Figure 1C. Most LAIs (165 of 228) were spontaneously active when recorded in current-clamp mode without current injection, as reported previously (Bennett and Wilson, 1999; Blomeley and Bracci, 2005). In 98 of 165 of these spontaneously active LAIs, intermittent quiescent periods $(>0.5 \mathrm{~s})$ were observed in which the membrane potential tended to hyperpolarize first and then depolarize until spike threshold was reached again. The negative peak of the membrane potential during these quiescent periods was on average $59 \pm 4 \mathrm{mV}$. In the remaining LAIs, which were not spontaneously active, the average resting membrane potential was $-57 \pm 5 \mathrm{mV}$. MSNs were also identified thanks to their distinctive electrophysiological properties, which included (1) strongly asymmetric responses to negative and positive current injections of the same magnitude, (2) slow depolarizing ramps observed during subthreshold depolarizations, and (3) delayed appearance of action potentials during depolarizing steps (Nisenbaum and Wilson, 1995; Bracci et al., 2004). An example of these features is shown in Figure $1 D$. The average resting membrane potential of MSNs was $-79 \pm 11 \mathrm{mV}$. 
A
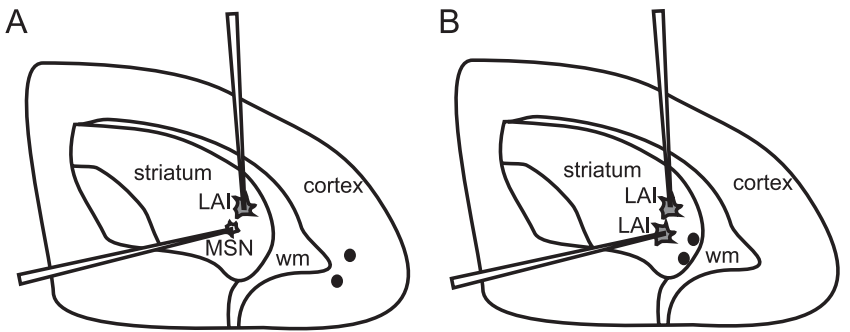

C

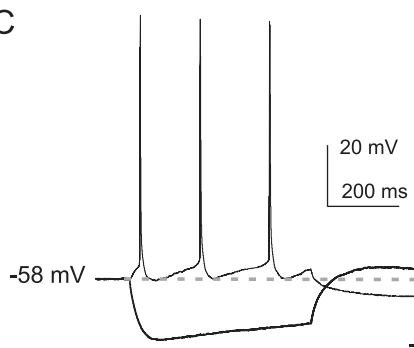

D
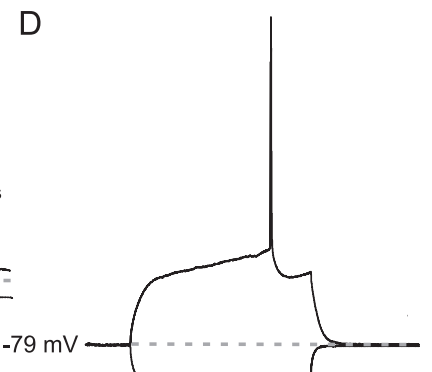

LAI

Figure 1. Positioning of recording and stimulating electrodes in oblique brain slices and typical electrophysiological features of an LAI and an MSN. $\boldsymbol{A}$, To investigate the effects of LAI spikes on glutamatergic input to MSN, the bipolar stimulating electrode (represented as 2 black circles) was placed in the medial agranular cortex, close to the white matter (wm), in oblique slices cut as described by Kawaguchi et al. (1989). Whole-cell recordings were obtained from an LAI (dark gray) and an MSN (light gray) in the dorsorostral portion of the striatum. Cell sizes are exaggerated for clarity. $\boldsymbol{B}$, To investigate the effects of LAI spikes on glutamatergic input to another LAl, the bipolar stimulating electrode was placed in the striatum. Whole-cell recordings were obtained from two LAls (dark gray). C, Membrane potential changes induced in an LAI by current injections ( +30 and $-180 \mathrm{pA}$ ); typical features of responses include a depolarizing sag that develops during the hyperpolarizing step, a rebound depolarization after the end of this step, and a long-lasting afterhyperpolarization after a train of spikes. No current was injected before and after the steps. Dashed gray line indicates resting membrane potential. $D$, Responses of an MSN to current injections ( $\pm 100 \mathrm{pA}$ ); the typical waveforms observed are characterized by a much larger voltage deflection in response to the depolarizing step, a slow depolarizing ramp that develops during the depolarizing step, and the delayed appearance of an action potential. No current was injected before and after the steps. Dashed gray line indicates resting membrane potential.

\section{Effects of LAI spikes on MSN glutamatergic responses}

The interaction between LAIs and MSNs was tested in 116 pairs located in the dorsolateral striatum at relative distances $<100$ $\mu \mathrm{m}$. Spontaneously active LAIs were slightly hyperpolarized by current injection (see Materials and Methods). We tried to elicit direct cholinergic responses in MSNs by triggering up to 20 spikes in the LAIs with current steps $(0.2-1.0 \mathrm{~s})$. However, in all pairs recorded, we failed to observe measurable responses in MSNs recorded in either current-clamp (at resting membrane potential) or voltage-clamp (holding potential range of -60 to -90 $\mathrm{mV}$ ) conditions. An example of this phenomenon is shown in Figure $2 A$. In 76 pairs, we triggered bursts of 5-10 spikes in the LAI while the MSN was voltage clamped from the holding value $(-80 \mathrm{mV})$ to levels between -100 and $+10 \mathrm{mV}$ (in $10 \mathrm{mV}$ increments; step duration of $0.5 \mathrm{~s}$ ). However, we could not reveal any significant differences in the membrane currents observed at any membrane potential level in the absence or the presence of LAI spikes. The MSN input resistance at $-80 \mathrm{mV}$, which was monitored in voltage clamp with $2-4 \mathrm{mV}$ steps, was also unaffected by LAI spikes.

We then investigated whether action potentials in the LAIs affected glutamatergic input to MSNs. In these experiments, a single electrical stimulus was applied to the cortex (as described

in Materials and Methods) every $15 \mathrm{~s}$ because, at this frequency, the EPSCs evoked in voltage-clamped MSNs or LAIs did not display any systematic trend of depression or potentiation (see Materials and Methods). Single action potentials were elicited in the LAI (recorded under current-clamp conditions) at variable intervals before an electrical stimulus. Evoked EPSCs were recorded from MSNs (under voltage-clamp conditions). Baseline EPSCs were recorded in MSNs in the absence of LAI spikes.

In 84 of 248 pairs tested (34\%), the presence of a spike in the LAI significantly reduced the amplitude of MSN responses for at least one of the intervals tested. An example of this phenomenon is illustrated in Figure 2D. The top traces are from an LAI and illustrate the timing of the evoked action potential with respect to the evoked synaptic responses (for clarity, only one trace for each interval tested is shown). The bottom traces are transmembrane currents from a simultaneously recorded MSN (voltage clamped at $-80 \mathrm{mV}$ ). For each interval, 10 consecutive current traces are superimposed. The current injections were timed so that the interval between the peak of the spike in the LAI and the peak of the EPSC in the MSN was $\sim 10,20,30,40$, or 60 ms. MSN responses recorded 10-40 ms after the LAI spike were significantly smaller than the control ones, whereas no significant difference was observed at $60 \mathrm{~ms}$. Maximal depression of evoked EPSCs was observed for $20 \mathrm{~ms}$ intervals in this pair. The plot in Figure $2 \mathrm{~B}$ illustrates the average effects observed in 13 pairs in which significant depression of EPSCs was observed, and at least three different intervals were tested. The average effects for all cases in which a significant reduction was observed for at least one interval are plotted in Figure 2C. Significant EPSC depression was observed in these pairs for intervals ranging between 10 and $60 \mathrm{~ms}$. Maximal effects were observed for intervals of $20-30 \mathrm{~ms}$ (18 and 19\% average EPSC reduction, respectively). The maximal reduction in MSN EPSC amplitude induced by an LAI spike ranged between 6 and $27 \%$ in the pairs tested.

Having observed that a single spike in an LAI depressed the glutamatergic input into a subset of neighboring MSNs, we tested whether a burst of spikes in an LAI could be more effective than a single one and/or affect a larger fraction of MSNs. We used current steps (100-120 ms long) to evoke trains of 5-12 spikes in LAIs, and we evoked EPSCs in neighboring MSNs 0-20 ms after the end of the current step. In all cases in which a single LAIs spike had no significant effects on MSN EPSC for intervals between 20 and $30 \mathrm{~ms}$, a train of LAI spikes also failed to elicit significant effects $(n=10)$. In 17 pairs in which a significant MSN EPSC reduction was observed with a single LAI spike, increasing the number of spikes preceding the EPSC to 5-10 did not cause an additional decrease in the EPSC amplitude, and, in four pairs, the inhibitory effects were significantly smaller than with a single spike. A representative example of these experiments is illustrated in Figure 3A. These observations suggested that acetylcholine release from LAI terminals may undergo substantial activitydependent depression (see Discussion).

We also tested how the acetylcholinesterase inhibitor neostigmine affected the ability of LAIs to suppress glutamatergic input to MSNs. Bath application of neostigmine bromide $(10 \mu \mathrm{M})$ per se (i.e., in the absence of evoked spikes in a neighboring LAI) caused a significant decrease in evoked MSN EPSC amplitude in 10 of 12 cases (Fig. $3 B$ ). On average, this reduction was $18 \pm 7 \%$. In seven pairs in which an LAI spike significantly reduced MSN EPSC amplitude in control solution, this reduction persisted in the presence of neostigmine but was significantly decreased, as shown in the example of Figure $3 B$. On average, EPSC inhibition was $25 \pm 6.8 \%$ in control solution and $14 \pm 6 \%$ in the presence of 
neostigmine. In five pairs in which an LAI spike did not significantly affect MSN EPSC, the same lack of effects was also observed after bath application of neostigmine.

\section{Effects of LAI spikes on LAI glutamatergic responses}

We also investigated the interaction between pairs of LAIs. We recorded 64 such pairs at relative distances $<200 \mu \mathrm{m}$. This distance (larger than for LAI-MSN pairs) was chosen because the scarcity of LAIs made it difficult to find a sufficient number of pairs at shorter distances. Direct synaptic connections between LAIs were tested by eliciting spikes in one LAI while recording from the other one in currentor voltage-clamp conditions, as described for LAI-MSN pairs. Such connections were not detectable in any of the pairs tested, as shown in the example of Figure $4 A$. We then tested the ability of LAI spikes to reduce evoked responses in another LAI. The protocol used was similar to the one described for LAI-MSN pairs, but electrical stimulation was delivered within the striatum every $15 \mathrm{~s}$.

A single spike in an LAI significantly depressed evoked EPSCs in a neighboring LAIs in 26 of 64 pairs (41\%). A representative example of these experiments is illustrated in Figure 4D. Top traces are from a current-clamped LAI in which spikes were evoked with short current injections; bottom traces are from a neighboring voltage-clamped LAI in which EPSCs were evoked at different intervals (10 consecutive current traces are superimposed for each interval). In this experiment, spike-EPSC intervals of $\sim 10,20,30$, and $40 \mathrm{~ms}$ were tested. Significant reduction of EPSC amplitude was present for 10-30 ms intervals. The average dependence of EPSC depression on the interval between the spike and the EPSC in nine pairs in which significant effects were observed and at least three intervals were tested is plotted in Figure $4 B$. The average effects for all cases in which a significant reduction was observed are plotted in Figure $4 C$. The time course of the EPSC depression in LAIs was broadly similar to the one observed in MSNs; significant effects were observed for intervals between 10 and $60 \mathrm{~ms}$, and maximal EPSC reduction was observed for intervals between 20 and $30 \mathrm{~ms}$ (on average 15\% for both intervals). Maximal depression across the pairs tested ranged between 8 and 19\% of control.

We also tested whether an individual LAI could affect its own glutamatergic input. To do this, we monitored (under currentclamp conditions) the EPSPs evoked at variable intervals after a single spike induced by a current injection. Examples of this kind of experiment are illustrated in Figure 5. A technical problem associated with this protocol is that, at short intervals after the spike, the evoked EPSPs were distorted by the decay phase of the offset transient from the depolarizing current pulse that triggered the spike; furthermore, these EPSPs were likely to be affected by postsynaptic events independent of acetylcholine release, such as the spike afterhyperpolarization typical of LAIs (Bennett et al., 2000). The EPSP amplitude was significantly reduced for inter-
B

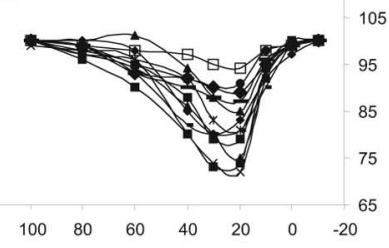

C
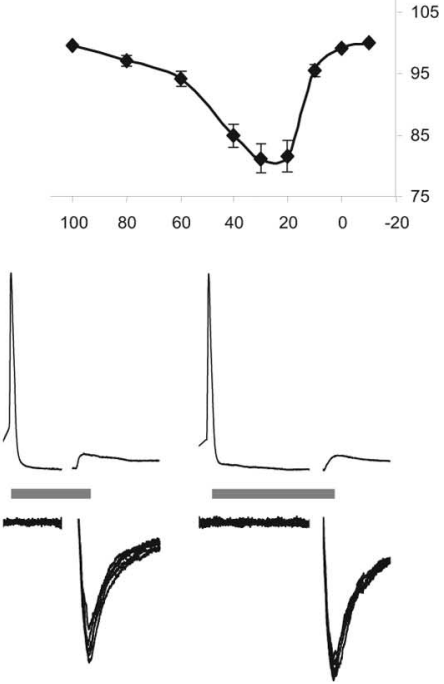
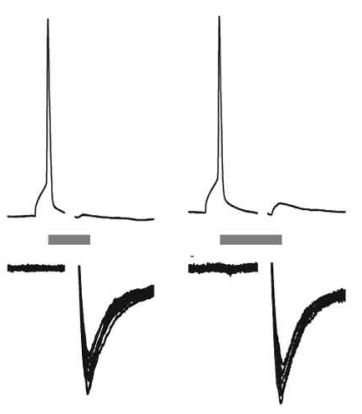

Figure 2. A single spike in an LAI inhibits evoked EPSC in a neighboring MSN. A, Simultaneous current-clamp recordings illustrate the lack of detectable effects of spikes in an LAI on the membrane potential of a neighboring MSN. The MSN was kept at

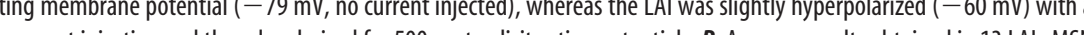
the origin in this and in the next figure. A positive value of the interval indicates that the LAI spike preceded the different intervals after the LAI spike. Gray bars represent the interval between the LAI spike peak and the MSN EPSC peak ( $\sim 10$, $20,30,40$, and $60 \mathrm{~ms})$. Electrical stimulation artifacts were removed in this and subsequent figures.

vals $<40 \mathrm{~ms}$ in 56 of 56 LAIs tested, whereas for intervals $\geq 40 \mathrm{~ms}$, a significant reduction was observed in 35 of 56 interneurons tested. The average time course of EPSP depression after a spike for 14 cases in which at least three different intervals were tested is illustrated in Figure 5A. The average effects for all LAIs in which a significant reduction was observed are plotted in Figure $5 B$. Maximal average reduction (18\%) was observed for $30 \mathrm{~ms}$ intervals. A representative example of these experiments is illustrated in Figure $5 C$, in which three spike-EPSP intervals (10, 20, and $40 \mathrm{~ms}$ ), in addition to baseline EPSPs, are presented. These results per se did not allow us to draw conclusions about whether a spike in an LAI affected the subsequent evoked EPSP through synaptic mechanisms. However, pharmacological experiments described below allowed us to dissect out these effects.

\section{Pharmacology of the effects of LAI spikes on evoked glutamatergic responses}

Exogenous activation of $\mathrm{M}_{2}$ and $\mathrm{M}_{3}$ receptors causes presynaptic inhibition of glutamatergic inputs to striatal MSNs (Sugita et al., 1991; Hernandez-Echeagaray et al., 1998). To test whether the present effects were also mediated by muscarinic receptors, we performed pharmacological experiments in LAI-MSN pairs in which a significant reduction was observed in control solution. A single spike was elicited in the LAI and, after a fixed delay, an EPSC was evoked in a neighboring $(<100 \mu \mathrm{m}) \mathrm{MSN}$. Intervals of 20-30 ms were used to maximize the inhibitory effects based on the results illustrated in Figures 2 and 3. Either atropine (a broadspectrum muscarinic antagonist; $20 \mu \mathrm{M}$ ) or methoctramine (at 

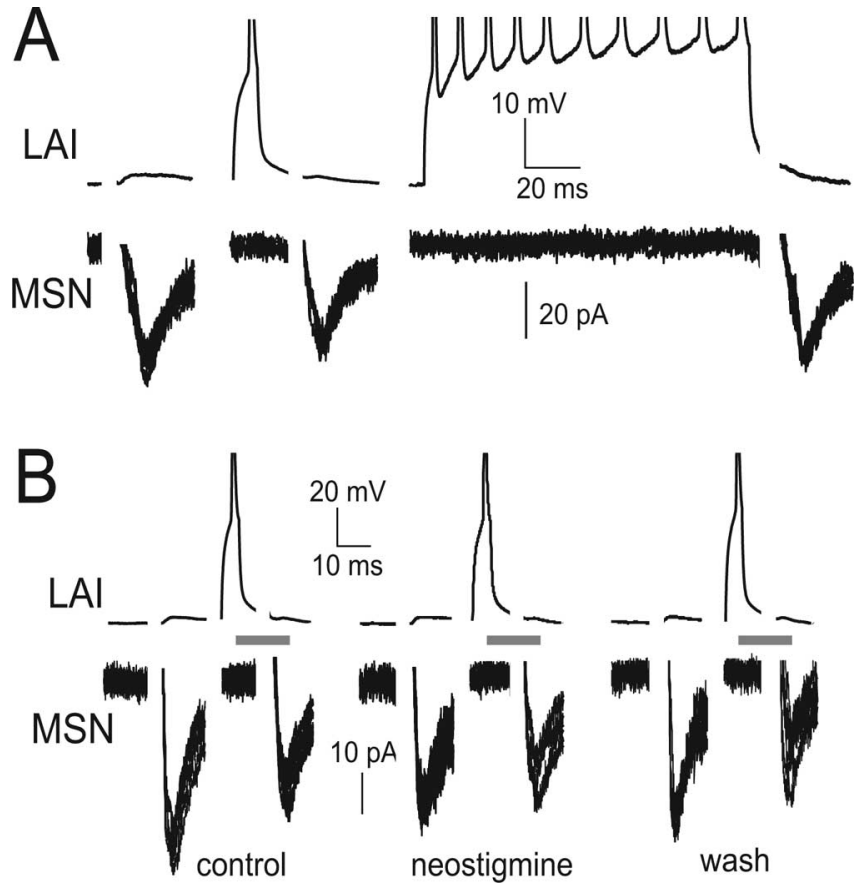

Figure 3. Effects of multiple spikes and neostigmine on EPSC inhibition. $A$, In this LAI-MSN pair, a single spike in the LAl significantly reduced the MSN EPSC amplitude for intervals of $\sim 25$ ms (left and middle traces; $10 \mathrm{MSN}$ responses are superimposed for each experiment illustrated in this figure; LAl spikes are truncated). Longer depolarizing current steps elicited 10 spikes and were timed so that the last spike occurred 20-25 ms before the peak of the evoked EPSC (right traces). The MSN EPSC amplitude after 10 LAI spikes was significantly larger than the one after a single spike. $\boldsymbol{B}$, In a different LAI-MSN pair, a single spike in the LAI significantly reduced MSN EPSC amplitude (interval of $20 \mathrm{~ms}$ ). In the presence of neostigmine, the MSN EPSC amplitude in the absence of LAl spikes was significantly decreased, and the inhibitory effects of an LAI spike were strongly reduced (from $27 \pm 4$ to $15 \pm 3 \%$ ). These effects were partially reversed on neostigmine washout.

$10 \mu \mathrm{M}$, a concentration that is expected to block both $\mathrm{M}_{2}$ and $\mathrm{M}_{3}$ muscarinic receptor) were subsequently bath applied. We found that bath application of atropine or methoctramine blocked the ability of LAIs to inhibit EPSCs in MSNs in all pairs tested ( $n=$ 24). This phenomenon is illustrated in the example of Figure $6 \mathrm{~A}$. In control solution, the EPSCs evoked in the MSN were significantly depressed by a preceding spike in the LAI for an interval $\sim 30 \mathrm{~ms}$ (in this experiment, the stimulation did not elicit detectable responses in the LAI). These effects were fully blocked by atropine because, in the presence of this drug, the MSN responses with or without spikes in the LAI were not significantly different.

Spike-induced inhibition of EPSC reappeared on washout. The average effects observed with this protocol are shown in Figure $6 B$ (results for atropine and methoctramine were grouped because no significant differences were observed in the effects of the two drugs). Eighteen of 24 experiments lasted enough for washout of the antagonists and reversal of their effects. Application of atropine or methoctramine also significantly increased baseline (i.e., with no spike evoked in the LAI) EPSC amplitude in 21 of $24 \mathrm{MSN}$ (as in the example of Fig. 6A).

Similar results were found for 13 pairs of LAIs (for these experiments, distance was $<200 \mu \mathrm{m}$ and the stimulator was placed in striatum). This phenomenon is illustrated in the example of Figure $7 A$. In control solution, a spike in an LAI caused significant reduction of EPSC amplitude in another LAI (interval of $\sim 20 \mathrm{~ms}$ ). Application of methoctramine suppressed this spikeinduced reduction, which reappeared on washout. As in the MSN case, bath application of atropine or methoctramine caused a significant increase in baseline EPSC amplitude in 10 of 13 LAIs. The average results for the effects of atropine or methoctramine (grouped together because no significant differences were found) are presented in Figure $7 B$.

To ascertain that the effects of atropine were not attributable to nonspecific effects on receptors different from the muscarinic ones, we performed additional experiments in which a much lower dose (500 nM) was used. In all cases in which significant effects of LAI spikes on the EPSC were observed in control solution in neighboring MSNs $(n=4)$ and LAIs $(n=4), 500 \mathrm{nM}$ atropine fully blocked these effects.

In the case of the self-induced depression of EPSPs in LAIs, we found that the effects of atropine or methoctramine on EPSP inhibition for spike-EPSP intervals up to $30 \mathrm{~ms}$ were variable and reached statistical significance only in 9 of 23 cases. However, when a $40 \mathrm{~ms}$ interval was used, atropine or methoctramine significantly reduced EPSP inhibition in 18 of 23 cases. This is illustrated in the example of Figure $8 A$, and the average effects observed with atropine or methoctramine are shown in Figure $8 B$. Thus, part of the EPSP reduction observed after a spike in LAIs was attributable to the activation of muscarinic receptors, presumably located on the glutamatergic terminals impinging on these cells.

Consistent with the experiment described above, application of atropine or methoctramine significantly increased the amplitude of baseline EPSPs in 16 of 23 cases.

Significant effects of LAI spikes on EPSCs were observed in subsets of LAI-MSN and LAI-LAI pairs. This may reflect the fact that only a fraction of MSNs and LAIs have their glutamatergic input controlled by muscarinic receptors. To test this hypothesis, we performed single recordings from MSNs and LAIs; evoked glutamatergic responses were monitored in control solution and after application of muscarine. Evoked EPSC amplitude was significantly reduced by muscarine in 12 of 12 MSNs (on average to $76.4 \pm 7.8 \%$ of control) and 10 of 11 LAIs (to $81.5 \pm 6.3 \%$ of control). These results show that most striatal neurons have their glutamatergic input inhibited by muscarinic receptors and suggests that a certain LAI controls glutamatergic input to a fraction of its neighboring cells.

\section{Effects of low extracellular potassium}

The observation that muscarinic blockers increased the amplitude of glutamatergic responses in MSNs and LAIs suggests that the spontaneous activity of some LAIs (different from the recorded ones, in which spontaneous firing activity was absent) inhibited glutamatergic terminals. However, another possible explanation is that these ligands acted as inverse agonists at muscarinic receptors (Costa and Cotecchia, 2005). To discriminate between these possibilities, we suppressed the spontaneous activity of LAIs by reducing extracellular potassium from 2.5 to $0.5 \mathrm{~mm}$. Under these conditions, which are expected to hyperpolarize neurons, spontaneous spikes completely disappeared in 15 of 21 LAIs recorded in current clamp without current injection. Low potassium solution caused a significant increase in the amplitude of baseline EPSCs recorded from MSNs in 17 of 21 cases, as shown in the example of a Figure $9 A$ (compare first EPSC in control solution and in low potassium).

The inhibitory effects of LAI spikes on MSN EPSC persisted in low potassium solution (Fig. 9A). In 21 of 21 pairs in which significant effects had been observed in control solution for intervals of 20-30 ms, LAI spikes still significantly decreased the MSN EPSC amplitude in low potassium. On average, spike- 
induced EPSC reduction was $12 \pm 3 \%$ in standard solution and $15 \pm 4 \%$ in low potassium solution. Average results for low potassium applications (with and without a spike in a neighboring LAI) are shown in Figure $9 B$.

Similar results were found for LAI-LAI interactions. Evoked EPSCs were significantly increased in low potassium solution in 10 of 11 LAIs tested. Furthermore, in 11 of 11 pairs, in which significant inhibition of an LAI EPSC was induced by a spike in another LAI in control solution, this inhibition persisted in low potassium solution. An example of this phenomenon is shown in Figure 9C, and average effects are presented in Figure 9D. On average, EPSC reduction in a neighboring LAI was $11 \pm 3 \%$ in standard solution and $15 \pm 2 \%$ in low potassium.

The effects of low potassium were occluded by previous application of atropine. In $12 \mathrm{MSN}$ and 15 LAIs, in which atropine significantly increased EPSC amplitude, subsequent application of low potassium failed to further increase the EPSCs. A representative example of these experiments is shown in Figure 9E.

Overall, these experiments indicate that glutamatergic input to MSNs and LAIs were affected by the spontaneous activity of LAIs.

\section{Discussion}

This is the first demonstration of how presynaptic inhibition depends on the action potentials of the neurons controlling the terminals. Single spikes in LAIs exerted a strong inhibitory control over the glutamatergic input to neighboring neurons. The time course of these cholinergic effects was fast, peaking 20-30 ms after the spike and disappearing after 40-80 ms. The maximal reduction in synaptic responses caused by a single LAI spike could be as large as $27 \%$ of the control amplitude in MSNs and 19\% in LAIs.

Glutamatergic terminals in the striatum are inhibited by exogenous application of muscarinic agonists (Malenka and Kocsis, 1988; Sugita et al., 1991; Calabresi et al., 1998b; Hernandez-Echeagaray et al., 1998). These effects are presynaptic and mediated by both $\mathrm{M}_{2}$ and $\mathrm{M}_{3}$ muscarinic receptors. Consistent with these findings, immunocytochemical studies revealed that $\mathrm{M}_{2}$ and $\mathrm{M}_{3}$ receptors are both present on corticostriatal terminals (Hersch et al., 1994). In the present study, the inhibitory effects caused by LAI action potentials were fully blocked by either atropine (a broadspectrum muscarinic antagonist) or by methoctramine, a muscarinic antagonist with some selectivity for $\mathrm{M}_{2}$ receptors but used here at a concentration that fully blocks both $\mathrm{M}_{2}$ and $\mathrm{M}_{3}$ recep-
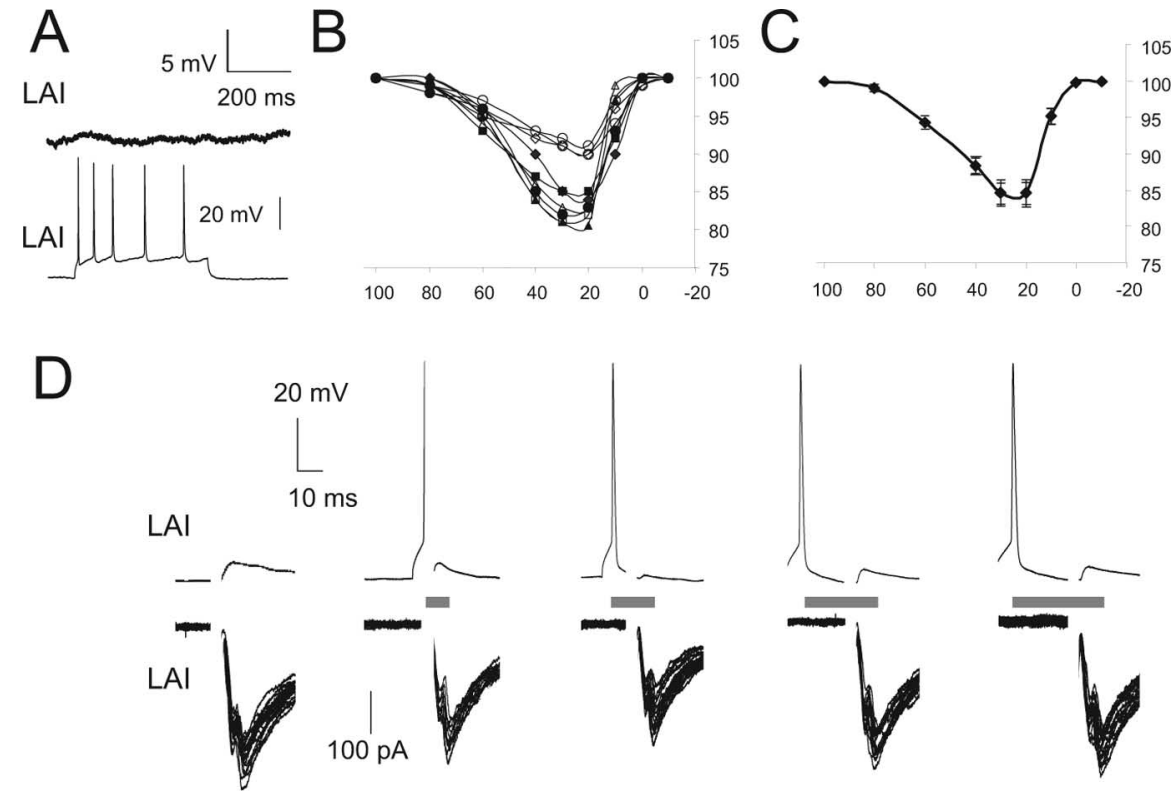

Figure 4. A single spike in an LAI inhibits evoked EPSC in a neighboring LAI. $\boldsymbol{A}$, An example of the lack of detectable direct interactions between two neighboring LAls. The two LAls were simultaneous recorded in current clamp and slightly hyperpolarized (at approximately $-60 \mathrm{mV}$ ) to prevent spontaneous firing; one of them was then depolarized for $500 \mathrm{~ms}$ to elicit action potentials. $\boldsymbol{B}$, Average effects of a single spike in an LAI on the evoked EPSC amplitude in a neighboring LAI. Data are from nine LAI-LAI pairs in which (1) significant effects were observed for some intervals and (2) at least three different intervals were tested. The EPSC amplitude (in percentage of control) is plotted as a function of the interval between the spike peak and the EPSC peak. C, Average effects of LAI spikes on neighboring LAI EPSC amplitude as a function of the interval, for all of the pairs in which significant effects were observed for at least one interval. $\boldsymbol{D}$, A representative example of the experiments performed to investigate these effects. Top traces are individual current-clamp recordings from an LAI, in which spikes (when present) were elicited with brief current injections at different intervals before an EPSC was evoked in a neighboring LAI. Bottom traces are superimposed recordings from the second $\mathrm{LAI}$ (voltage clamp; $V_{\mathrm{h}}=-60 \mathrm{mV}$ ), showing the EPSCs evoked at different intervals after the spike in the other LAI. Gray bars represent the intervals between the LAI spike peak and the other LAI EPSC peak ( $\sim 10,20,30$, and 40 ms).

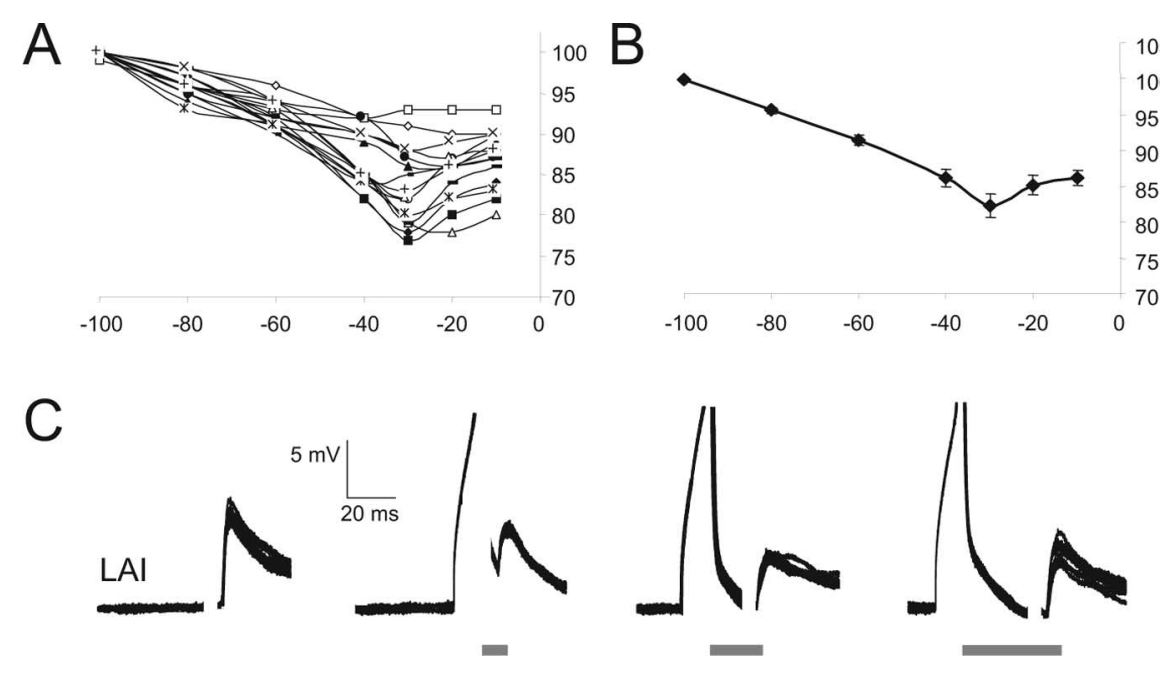

Figure 5. Effects of an LAI single spike on the EPSP evoked in the same cell. $\boldsymbol{A}$, Average effects of a single spike in an LAI on the amplitude of the evoked EPSPs recorded in the same neuron, as a function of the interval between the two events. Data are from 14 experiments in which at least three different intervals were tested. $\boldsymbol{B}$, Overall average effects for all of the cells in which significant effects were observed for at least one interval. $C$, A representative example of these experiments, in which an EPSP was evoked at variable times after an action potential was elicited in the same cell by a short current injection. Spikes are truncated. Gray bars represent the interval between the peak of the spike and that of the EPSP $(\sim 10,20$, and $40 \mathrm{~ms})$.

tors (Budriesi et al., 2001; Pujol Lereis et al., 2006). This suggests that the receptors involved in these effects were the same activated exogenously in previous studies. We did not attempt to further dissect the identity of the muscarinic receptors mediating these effects. Because of the poor selectivity of available antago- 


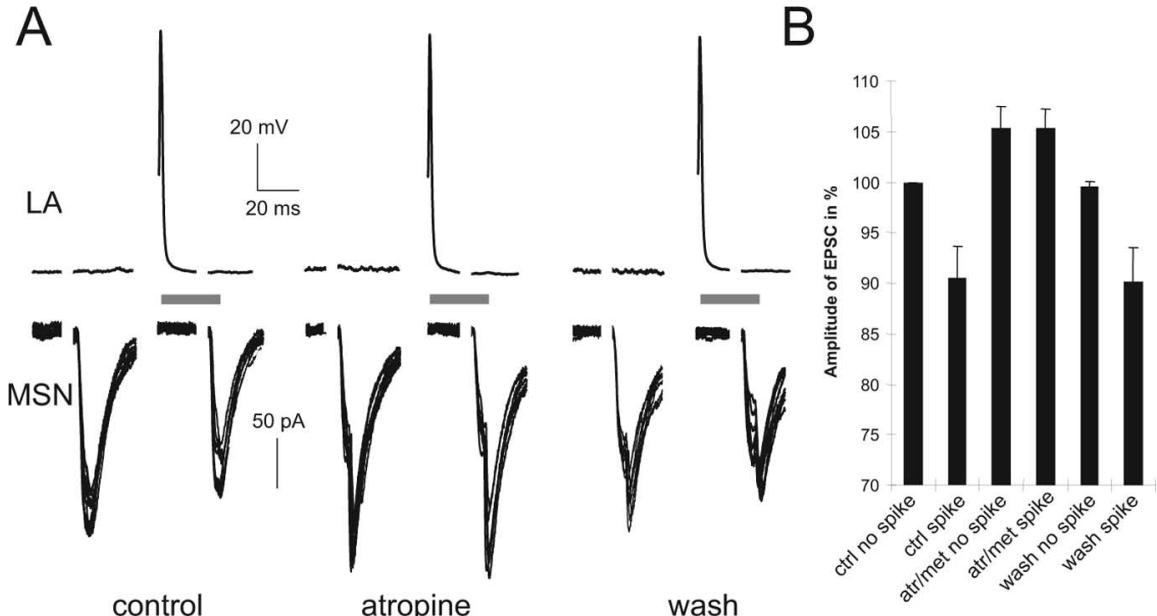

Figure 6. Spike-induced inhibition of evoked EPSCs in MSNs is mediated by muscarinic receptors. $\boldsymbol{A}$, Traces from a representative experiment in which significant MSN EPSC suppression was induced by an LAI spike in control solution, for an interval of $\sim 30$ ms (gray bars). In the presence of atropine, the baseline MSN EPSC amplitude was significantly increased, and a spike in the LAI no longer affected such EPSC. These effects were reversed on atropine washout. $\boldsymbol{B}$, Average effects of application of atropine or methoctramine in 24 LAI-MSN pairs, in which significant spike-induced suppression of MSN EPSC was observed in control solution. The spike-EPSC interval was constant for each pair (range of $20-30 \mathrm{~ms}$ ).

A

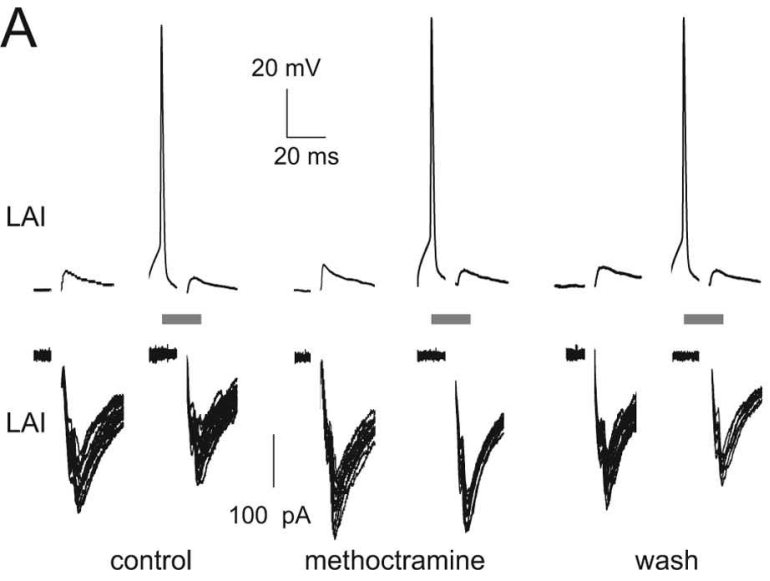

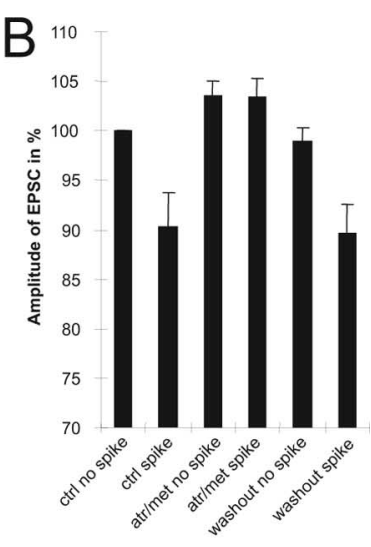

Figure 7. Spike-induced inhibition of evoked EPSC in LAl is mediated by muscarinic receptors. $\boldsymbol{A}$, A representative experiment in which significant EPSC suppression was induced in an LAI by a spike generated by a neighboring LAl in control solution (interval of $20 \mathrm{~ms}$; gray bars). In the presence of methoctramine, the LAI EPSC amplitude was significantly increased, and a spike in the other LAI no longer affected this EPSC. These effects were reversed on methoctramine washout. $\boldsymbol{B}$, Average effects of application of atropine or methoctramine in $13 \mathrm{LAI}-\mathrm{LAI}$ pairs, in which significant spike-induced suppression of EPSC was observed in control solution. The spike-EPSC interval (range of $20-30 \mathrm{~ms}$ ) was constant for each pair.

nists for the muscarinic receptor subtypes, this will require the use of dose-response curves to measure dissociation equilibrium constants of different agents (Sugita et al., 1991; Pujol Lereis et al., 2006).

In the present study, acetylcholine was synaptically released after a spike, and presumably its concentration rapidly decreased to baseline level. Therefore, it was not possible to use paired-pulse protocols, which are usually performed to test the presynaptic or postsynaptic site of action of an agonist when its concentration is constant. Nevertheless, our results are fully consistent with a presynaptic action of acetylcholine on glutamatergic terminals. The currents observed in MSNs at different voltage levels, and the input resistance of these cells, were unaffected by spikes in a neighboring LAI, despite the fact that several postsynaptic effects are observed with application of exogenous agonists (Calabresi et al., 2000; Zhou et al., 2003; Shen et al., 2005). This may be explained by two considerations. First, the receptors mediating cholinergic postsynaptic effects have much lower affinity for their agonists than the presynaptic ones (Barral et al., 1999). Synaptically released acetylcholine may not have reached concentrations high enough for significant postsynaptic receptor activation. Furthermore, the intracellular dialysis caused by conventional whole-cell recordings may have interfered with postsynaptic metabolic processes; in a recent study, perforated patch techniques were used to reveal the effects of $M_{1}$ receptor activation on KCNQ potassium currents in MSN, because these currents and their muscarinic modulation are easily disrupted by intracellular dialysis (Shen et al., 2005). Given this absence of detectable postsynaptic effects, we can rule out that the EPSC reduction observed in the present study was attributable to muscarinic modulation of some MSN conductances (involving for instance a decrease in input resistance). These effects are also unlikely to be attributable to a direct action on ionotropic glutamate receptors, because previous studies have shown that muscarinic agonist enhance, rather than depress, NMDA receptor activity and do not affect AMPA receptors (Calabresi et al., 1998a). Such effects on NMDA receptors were not apparent in our experiments, which were performed at negative membrane potentials at which activation of NMDA receptors is strongly limited by magnesium blockade (Mayer et al., 1984).

The inhibitory effects of a train of 5-10 LAI spikes was either similar or smaller than those of a single spike. This could be caused by strong activity-dependent depression of acetylcholine release, so that its concentration in proximity of glutamatergic terminals did not increase during a burst of spikes. This kind of depression has been observed at GABAergic synapses between two MSNs and between a GABAergic interneurons and an MSN, in which the average response to the first spike in a burst is two to five times larger than that elicited by subsequent spikes (Koos et al., 2004).

The inhibitory effects of LAI spikes on evoked glutamatergic potentials were broadly similar in neighboring MSNs and LAIs. Thirty-four percent of MSNs located within $100 \mu \mathrm{m}$ from an LAI and $41 \%$ of LAIs located within $200 \mu \mathrm{m}$ had their glutamatergic input inhibited by this neuron. Because recordings were made from cells located on the surface of the slice, in which some neuronal processes are inevitably severed, it seems likely that connectivity would be larger (at least by a factor of two) in the intact tissue. Pairs of neighboring MSNs are directly connected in $\sim 17 \%$ of cases (Koos et al., 2004), whereas GABAergic interneurons contact $\sim 25 \%$ of MSNs located within $250 \mu \mathrm{m}$ (Koos and Tepper, 1999). We investigated pairs located at smaller distances. 
However, in these previous studies, the presence of connections did not depend on distance within the investigated range. The present data show that cholinergic control of glutamatergic input is another major form of synaptic communication in the striatum. LAIs are $<1 \%$ of the striatal neuronal population, but they have a dense and relatively large axonal arborization (Bolam et al., 1984), so that their anatomy is consistent with a prominent control of the local network.

Atropine and methoctramine increased baseline EPSC amplitude in most MSNs and LAIs. This suggested that inhibition of the glutamatergic terminals was present in the slice because of the spontaneous activity of some LAIs. However, an alternative explanation was that these ligands acted as inverse agonists at muscarinic receptors on glutamatergic terminals (Costa and Cotecchia, 2005). To discriminate between these two hypotheses, we used low potassium, which was effective in silencing spontaneously active LAIs but did not interfere directly with muscarinic receptors. Low potassium did not block the inhibitory effects of LAI spikes on evoked EPSC but increased baseline EPSCs in most MSNs and LAIs, and this effect was occluded by previous application of muscarinic antagonists. This shows that acetylcholine released by spontaneously active LAIs did limit baseline EPSC and that, in most cases, at least another LAI (in addition to the recorded one) exerted some inhibitory influence on glutamatergic terminals. The EPSC increase induced by low potassium or muscarinic agonists was relatively small when compared with the effects of a single spike in the recorded LAI; this may suggest that, on average, only one LAI (besides the recorded one) affected the glutamatergic input to a given cell. It is difficult, however, to draw firm conclusions on this, because the rate of spontaneous spike generation in these cells is quite variable (Bennett and Wilson, 1999; Blomeley and Bracci, 2005), and receptor desensitization may limit responses to prolonged release of acetylcholine (Hosey et al., 1995; Sokolova et al., 2005).

The presence of a tonic cholinergic inhibition of glutamatergic terminals was also demonstrated by the fact that the acetylcholinesterase inhibitor neostigmine decreased EPSC amplitude. Under these conditions, the effects of the spike evoked in an LAI were reduced, presumably because a significant fraction of presynaptic muscarinic receptors were already activated by acetylcholine released by other LAIs. It is possible that doses of neostigmine much smaller than that used here would increase the
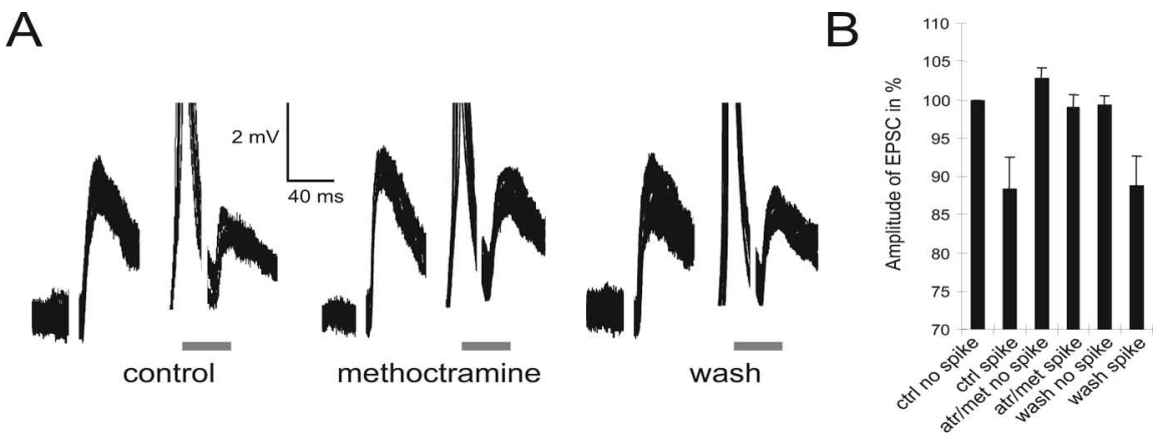

Figure 8. Afterspike reduction of evoked EPSPs in LAls is partly mediated by muscarinic receptors. $A$, A representative experiment showing the effects of methoctramine on afterspike reduction of evoked EPSPs in an LAI. In control solution, an EPSP was evoked after a spike was triggered in the same cell; spike-EPSP interval was $\sim 40$ ms (gray bars; the spikes are truncated in these traces). In the presence of methoctramine, the EPSP after a spike is still significantly smaller than the one evoked without a preceding spike, but the spike-induced inhibition is significantly reduced. These effects are reversed on washout. $\boldsymbol{B}$, Average effects on afterspike reduction of EPSP in 23 LAls in which either atropine or methoctramine were applied.
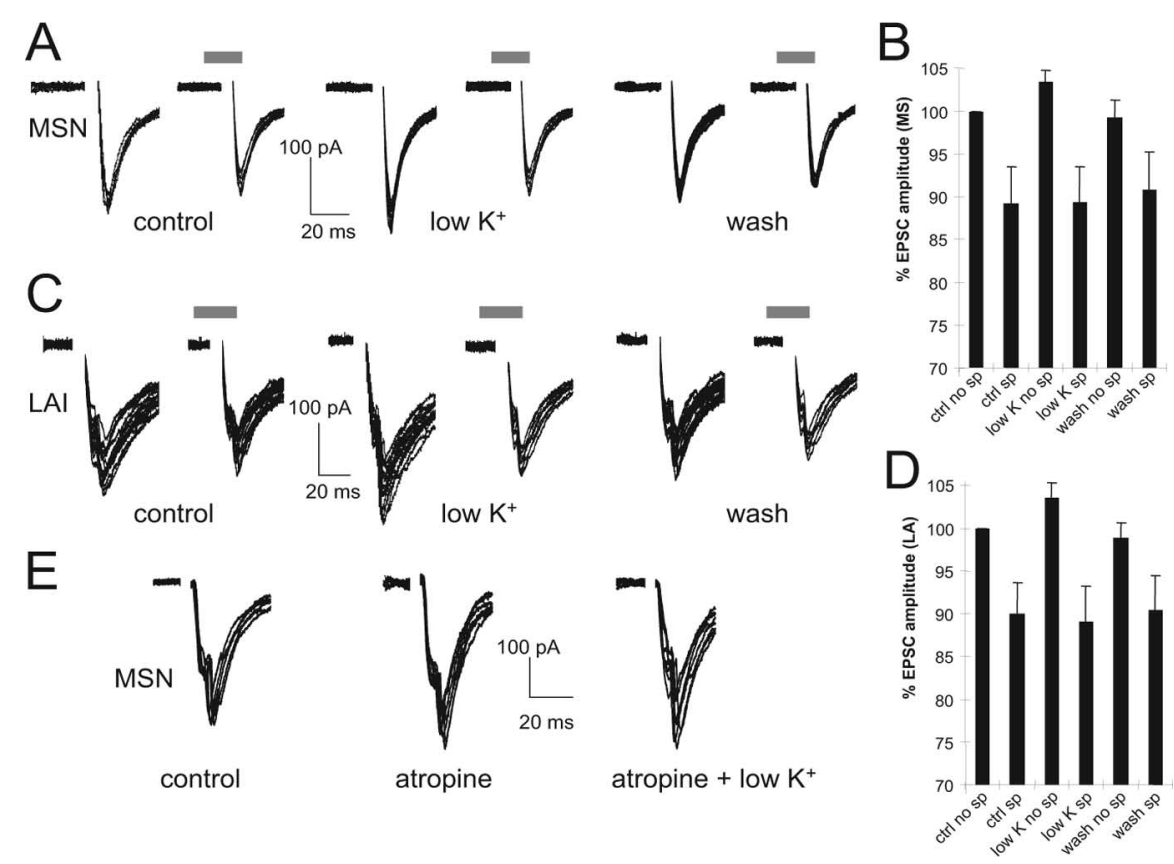

Figure 9. Effects of low potassium on evoked EPSCS and their spike-induced modulation. $\boldsymbol{A}$, In this representative experiment, paired recordings were obtained from an LAI and an MSN. Only the MSN synaptic currents are shown (10 superimposed traces are presented for each condition), without a spike in the LAI (no gray bar) or with one (gray bars represent time between peak of spike and peak of EPSC). In control solution, a spike in the LAI significantly decreased the amplitude of the evoked EPSC in the MSN (at an interval of $\sim 20 \mathrm{~ms}$ ). In the presence of low potassium solution, the EPSC amplitude in the absence of an LAI spike was significantly increased, and the inhibitory effects of a spike in the neighboring LAI were still present. These effects were reversed on washout. $\boldsymbol{B}$, Average effects of low potassium solution on MSN EPSC amplitude in the absence, or presence, of a spike in a neighboring LAI. C, Traces form a paired recording experiment from two LAls. In control solution, a spike in one LAI (recorded in current-clamp mode) significantly decreased the amplitude of the evoked EPSC in the other LAI (recorded in voltage clamp). The spike-EPSC interval was $\sim 30 \mathrm{~ms}$. Superimposed traces from the LAl recorded in voltage clamp are shown, in the absence (no gray bar) or presence of a spike in the other LAI (in this case, gray bars represent time between peak of spike and peak of EPSC). In the presence of low potassium solution, the EPSC amplitude in the absence of a spike in the other LAI was significantly increased, and the inhibitory effects of a spike in the neighboring LAI were still present. These effects were reversed on washout. $\boldsymbol{D}$, Average effects of low potassium solution on LAI EPSC amplitude in the absence, or presence, of a spike in a neighboring LA. $\boldsymbol{E}$, In an MSN, evoked EPSCs were significantly increased in amplitude by atropine application. However, subsequent application of low potassium solution (still in the presence of atropine) failed to affect EPSC amplitude.

effects of an LAI spike without causing a significant increase of background inhibition.

In the intact brain, LAIs are much more active than in vitro, because they are tonically and phasically excited by a variety of 
neurotransmitters, including glutamate, dopamine, and serotonin (Wilson et al., 1990; Aosaki et al., 1998; Reynolds and Wickens, 2004; Blomeley and Bracci, 2005). Thus, the present data imply that, in vivo, glutamatergic input to MSNs and LAIs is subject to a strong inhibitory cholinergic control.

In awake animals, a marked pause in LAI firing is observed in response to salient or rewarding events (Cragg, 2006). Based on the present results, MSNs located in proximity of pausing LAIs will transiently become much more responsive to cortical inputs. When LAI firing resumes, it will cause an abrupt reduction of MSNs excitation, thus precisely limiting the temporal extent of their responses.

This transient increase of their cortical input will have immediate effects on the firing activity of these MSNs, thus shaping the motor response to the stimulus. Furthermore, increased corticostriatal responses in MSNs, and the resulting increased probability of their firing, will increase the likelihood that Hebbian longterm modification of corticostriatal synapses take place. This is accentuated by the fact that pauses in LAI firing coincide with sharp increases in dopamine neuron firing (Cragg, 2006), and dopamine release in the striatum is required for long-term plasticity (Reynolds and Wickens, 2002). A concomitant increase in dopamine and decrease in acetylcholine levels will thus create ideal conditions for long-term modification of corticostriatal synapses, which is thought to underlie reinforcement motor learning (Kelley et al., 2003; Gurney et al., 2004).

\section{References}

Aosaki T, Kiuchi K, Kawaguchi Y (1998) Dopamine $\mathrm{D}_{1}$-like receptor activation excites rat striatal large aspiny neurons in vitro. J Neurosci 18:5180-5190.

Aubert I, Cecyre D, Gauthier S, Quirion R (1996) Comparative ontogenic profile of cholinergic markers, including nicotinic and muscarinic receptors, in the rat brain. J Comp Neurol 369:31-55.

Aznavour N, Mechawar N, Descarries L (2002) Comparative analysis of cholinergic innervation in the dorsal hippocampus of adult mouse and rat: a quantitative immunocytochemical study. Hippocampus 12:206-217.

Barral J, Galarraga E, Bargas J (1999) Muscarinic presynaptic inhibition of neostriatal glutamatergic afferents is mediated by Q-type $\mathrm{Ca}^{2+}$ channels. Brain Res Bull 49:285-289.

Bennett BD, Wilson CJ (1999) Spontaneous activity of neostriatal cholinergic interneurons in vitro. J Neurosci 19:5586-5596.

Bennett BD, Callaway JC, Wilson CJ (2000) Intrinsic membrane properties underlying spontaneous tonic firing in neostriatal cholinergic interneurons. J Neurosci 20:8493-8503.

Blomeley C, Bracci E (2005) Excitatory effects of serotonin on rat striatal cholinergic interneurones. J Physiol (Lond) 569:715-721.

Bolam JP, Wainer BH, Smith AD (1984) Characterization of cholinergic neurons in the rat neostriatum. A combination of choline acetyltransferase immunocytochemistry, Golgi-impregnation and electron microscopy. Neuroscience 12:711-718.

Bracci E, Panzeri S (2006) Excitatory GABAergic effects in striatal projection neurons. J Neurophysiol 95:1285-1290.

Bracci E, Centonze D, Bernardi G, Calabresi P (2004) Engagement of rat striatal neurons by cortical epileptiform activity investigated with paired recordings. J Neurophysiol 92:2725-2737.

Budriesi R, Cacciaguerra S, Toro RD, Bolognesi ML, Chiarini A, Minarini A, Rosini M, Spampinato S, Tumiatti V, Melchiorre C (2001) Analysis of the muscarinic receptor subtype mediating inhibition of the neurogenic contractions in rabbit isolated vas deferens by a series of polymethylene tetra-amines. Br J Pharmacol 132:1009-1016.

Calabresi P, Centonze D, Gubellini P, Pisani A, Bernardi G (1998a) Endogenous ACh enhances striatal NMDA-responses via M1-like muscarinic receptors and PKC activation. Eur J Neurosci 10:2887-2895.

Calabresi P, Centonze D, Gubellini P, Pisani A, Bernardi G (1998b) Blockade of M2-like muscarinic receptors enhances long-term potentiation at corticostriatal synapses. Eur J Neurosci 10:3020-3023.
Calabresi P, Centonze D, Gubellini P, Pisani A, Bernardi G (2000) Acetylcholine-mediated modulation of striatal function. Trends Neurosci 23:120-126.

Contant C, Umbriaco D, Garcia S, Watkins KC, Descarries L (1996) Ultrastructural characterization of the acetylcholine innervation in adult rat neostriatum. Neuroscience 71:937-947.

Costa T, Cotecchia S (2005) Historical review: negative efficacy and the constitutive activity of G-protein-coupled receptors. Trends Pharmacol Sci 26:618-624.

Cragg SJ (2006) Meaningful silences: how dopamine listens to the ACh pause. Trends Neurosci 29:125-131.

Duvoisin RC (1967) Cholinergic-anticholinergic antagonism in parkinsonism. Arch Neurol 17:124-136.

Gurney K, Prescott TJ, Wickens JR, Redgrave P (2004) Computational models of the basal ganglia: from robots to membranes. Trends Neurosci 27:453-459.

Hernandez-Echeagaray E, Galarraga E, Bargas J (1998) 3-Alpha-chloroimperialine, a potent blocker of cholinergic presynaptic modulation of glutamatergic afferents in the rat neostriatum. Neuropharmacology 37:1493-1502.

Hersch SM, Gutekunst CA, Rees HD, Heilman CJ, Levey AI (1994) Distribution of m1-m4 muscarinic receptor proteins in the rat striatum: light and electron microscopic immunocytochemistry using subtype-specific antibodies. J Neurosci 14:3351-3363.

Hosey MM, Benovic JL, DebBurman SK, Richardson RM (1995) Multiple mechanisms involving protein phosphorylation are linked to desensitization of muscarinic receptors. Life Sci 56:951-955.

Kawaguchi Y (1993) Physiological, morphological, and histochemical characterization of three classes of interneurons in rat neostriatum. J Neurosci 13:4908-4923.

Kawaguchi Y, Wilson CJ, Emson PC (1989) Intracellular recording of identified neostriatal patch and matrix spiny cells in a slice preparation preserving cortical inputs. J Neurophysiol 62:1052-1068.

Kelley AE, Andrzejewski ME, Baldwin AE, Hernandez PJ, Pratt WE (2003) Glutamate-mediated plasticity in corticostriatal networks: role in adaptive motor learning. Ann NY Acad Sci 1003:159-168.

Koos T, Tepper JM (1999) Inhibitory control of neostriatal projection neurons by GABAergic interneurons. Nat Neurosci 2:467-472.

Koos T, Tepper JM, Wilson CJ (2004) Comparison of IPSCs evoked by spiny and fast-spiking neurons in the neostriatum. J Neurosci 24:7916-7922.

Lapper SR, Bolam JP (1992) Input from the frontal cortex and the parafascicular nucleus to cholinergic interneurons in the dorsal striatum of the rat. Neuroscience 51:533-545.

Malenka RC, Kocsis JD (1988) Presynaptic actions of carbachol and adenosine on corticostriatal synaptic transmission studied in vitro. J Neurosci 8:3750-3756

Mayer ML, Westbrook GL, Guthrie PB (1984) Voltage-dependent block by $\mathrm{Mg}^{2+}$ of NMDA responses in spinal cord neurones. Nature 309:261-263.

Miles R, Poncer JC (1996) Paired recordings from neurones. Curr Opin Neurobiol 6:387-394.

Nicoll RA, Malenka RC, Kauer JA (1990) Functional comparison of neurotransmitter receptor subtypes in mammalian central nervous system. Physiol Rev 70:513-565.

Nisenbaum ES, Wilson CJ (1995) Potassium currents responsible for inward and outward rectification in rat neostriatal spiny projection neurons. J Neurosci 15:4449-4463.

Phelps PE, Houser CR, Vaughn JE (1985) Immunocytochemical localization of choline acetyltransferase within the rat neostriatum: a correlated light and electron microscopic study of cholinergic neurons and synapses. J Comp Neurol 238:286-307.

Pujol Lereis VA, Hita FJ, Gobbi MD, Verdi MG, Rodriguez MC, Rothlin RP (2006) Pharmacological characterization of muscarinic receptor subtypes mediating vasoconstriction of human umbilical vein. Br J Pharmacol 147:516-523.

Reynolds JN, Wickens JR (2002) Dopamine-dependent plasticity of corticostriatal synapses. Neural Netw 15:507-521.

Reynolds JN, Wickens JR (2004) The corticostriatal input to giant aspiny interneurons in the rat: a candidate pathway for synchronising the response to reward-related cues. Brain Res 1011:115-128. 
Shen W, Hamilton SE, Nathanson NM, Surmeier DJ (2005) Cholinergic suppression of KCNQ channel currents enhances excitability of striatal medium spiny neurons. J Neurosci 25:7449-7458.

Sokolova E, Matteoni C, Nistri A (2005) Desensitization of neuronal nicotinic receptors of human neuroblastoma SH-SY5Y cells during short or long exposure to nicotine. Br J Pharmacol 146:1087-1095.

Sugita S, Uchimura N, Jiang ZG, North RA (1991) Distinct muscarinic receptors inhibit release of gamma-aminobutyric acid and excitatory amino acids in mammalian brain. Proc Natl Acad Sci USA 88:2608-2611.

Tepper JM, Bolam JP (2004) Functional diversity and specificity of neostriatal interneurons. Curr Opin Neurobiol 14:685-692.

Turecek R, Trussell LO (2001) Presynaptic glycine receptors enhance transmitter release at a mammalian central synapse. Nature 411:587-590.
Wilson CJ (2005) The mechanism of intrinsic amplification of hyperpolarizations and spontaneous bursting in striatal cholinergic interneurons. Neuron 45:575-585.

Wilson CJ, Chang HT, Kitai ST (1990) Firing patterns and synaptic potentials of identified giant aspiny interneurons in the rat neostriatum. J Neurosci 10:508-519.

Wu LG, Saggau P (1997) Presynaptic inhibition of elicited neurotransmitter release. Trends Neurosci 20:204-212.

Zhou FM, Wilson CJ, Dani JA (2002) Cholinergic interneuron characteristics and nicotinic properties in the striatum. J Neurobiol 53:590-605.

Zhou FM, Wilson C, Dani JA (2003) Muscarinic and nicotinic cholinergic mechanisms in the mesostriatal dopamine systems. The Neuroscientist 9:23-36. 